\section{Insight into pathophysiology of sudden infant death syndrome}

JULIJE MEŠTROVIĆ ( $\square$ )

Paediatric Intensive Care Unit

Department of Paediatrics,

Split University Hospital

Spinčićeva 1, 21000 Split, Croatia

Phone: + 385 (0) 21556686

Fax: + $385(0) 21556590$

E-mail: julije.mestrovic@st.t-com.hr

\begin{abstract}
Physiological studies that have been undertaken in children with apparent life-threatening events point to disturbances of the autonomic nervous system and brain stem abnormalities. These abnormalities are manifested as breathing dysfunctions and sleeping patterns, as well as heart rate variability. The diminished arousal response in infants who are exposed to intermittent hypoxia can be fatal during conditions when oxygen availability is limited. This inference well explains well the success of campaigns for supine sleeping position, which associated with the decrease in the rate of sudden infant syndrome deaths.
\end{abstract}

Key words: apparent life-threatening event, sudden infant death syndrome, autonomic nervous system, sleep

Apparent life-threatening event (ALTE) is an episode that is frightening to the observer and is characterized by a combination of apnea (central or obstructive), color change (cyanotic or pallid, and sometimes plethoric), marked changes in muscle tone (usually limpness), choking and gagging. In some cases, the observer fears that the infant has died. This definition is intentionally broad leaving a wide variety of events which can be considered an ALTE. (1)

Previously used terminology such as "near-miss SIDS" should be abandoned because it implies a possibly misleading close association between this type of spell and sudden infant death syndrome (SIDS).

SIDS has been recognized as a diagnostic entity since 1969. It is defined as the sudden death of any infant that remains unexplained despite a complete postmortem investigation, including an autopsy, examination of the scene of death, and review of the case history. (2)

The relationship between ALTE and SIDS is controversial. The proposed relationship motivates physicians to approach ALTE as a potentially fatal entity. Most children with ALTE have a mild event and are probably at no increased risk of SIDS. Approximately $1-3 \%$ of all infants in the general population experience an ALTE. The estimated risk of SIDS in a population of infants with ALTE is $1 \%$. Of all infants who die from SIDS, $5-6 \%$ had a prior history of an ALTE. (3) However, there seem to be stronger data linking so called "serious" ALTE with an increased incidence of SIDS. The so called "serious" ALTE is defined as an infant who experiences an ALTE during sleep or when some form of CPR or vigorous stimulation is required. The group of infants who had more than one "serious" ALTE, had a $28 \%$ subsequent risk for SIDS. (4)

The ALTE population is heterogeneous, and the differential diagnosis of ALTE is broad (table 1). (3)

Apnea of infancy (AOI) is an unexplained episode of cessation of breathing for 20 seconds or longer, or a shorter respiratory pause associated with bradycardia, cyanosis, pallor and/or marked hypotonia. The terminology "apnea of infancy" generally refers to infants who are greater than 37 weeks gestational age at the onset of pathologic apnea. AOI should be reserved for those infants for whom no specific cause of ALTE can be identified. In other words, these are infants whose ALTE was idiopathic and believed to be related to apnea. (5)

Many physiological studies have been undertaken in children with $\mathrm{AO}$. The results of those studies point to the disturbances of the autonomic nervous system and brain stem abnormalities. Brain stem abnormalities include 
Table 1. Differential diagnosis of apparent life-threatening event.

Normal
Mild periodic breathing during sleep
Occasional 5-15 second central apnea during sleep
Occasional/rare mild choking with overaggressive sucking during feeds

\section{Acute Conditions}

Infection

Sepsis/meningitis

Respiratory syncytial virus

Pertussis

Drug induced

Other respiratory infections

Postanesthetic

\section{Chronic Conditions}

Gastroesophageal reflux

Seizure

Other neurologic disorders (e.g. CNS tumor)

Subdural hemorrhage

Arnold-Chiari associated apnea

Cardiac dysrhytmia

Abnormalities of respiratory drive

Immature respiratory center

Apnea of prematurity

Respiratory center dysfunction

Central hypoventilation syndrome (Ondine's course)

Obstructive sleep apnea

Vocal cord paralysis

Tracheomalacia

Vascular ring

Child abuse

Munchausen by proxy

Idiopathic

focal astrogliosis, persistent dendritic spines, and hypomyelination. (6) There is a significant correlation between the degree of apoptosis in the dorsal vagus nucleus in medulla oblongata and the frequency of obstructive apnea in SIDS victims. (7)

The prominent features of infants with AO include:

1. Breathing: more frequent obstructive apnea, and longer central apnea

2. Sleeping: less movements during sleep, longer duration of REM sleep, less wakening during sleep, diminished ventilatory responsiveness to hypercarbia and hypoxia

3. Heart: higher heart rate, reduced heart rate variation. $(3,8,9)$ three times higher in premature infants. The population of preterm infants who were exposed to maternal smoking in utero have increased frequent apnea, and marked decrease in the number of arousals, compared to premature infants who had not been exposed to cigarette smoke before birth. (12)

During the several recent years some important revelations have given new insight into the existing knowledge of respiratory control. The center for generation of respiratory rhythmic activity has been identified in the brainstem region. This zone, consists of between 150 and 200 neurons, can induce various respiratorypatternse.g. eupneic, gasping and sighing. (13) Contrary to previous concepttions, central chemosensitive neurons have been found not only in the ventral medullary surface, but located diffusely in the central nervous system. (14) Finally, it has been found that peripheral chemoreceptors are not the sole site where excitatory impulses to hypoxia are induced, since multiple regions of the brain can provoke increases in ventilation as the response to hypoxia. (15) The neural network responsible for response to hypoxia can modify its properties as a result of preceding experiences. (16) When applied to SIDS victims, the exposures to intermittent hypoxia might delay the arousal responses, and could be fatal during conditions when the oxygen availability is limited. (17)

In order to reduce the incidence of SIDS, several strategies have been introduced for populations that could be at greater danger. The risk for SIDS enhanced by maternal smoking has already been emphasized in this text. The strategy that has attained the biggest and the most sustained effect in various studies that have been pursued in various countries is the "back to sleep" campaign. The data from the USA show that the campaign for supine sleeping position was associated with the decrease in the rate of SIDS deaths from 1,20 deaths per 1000 live births in 1992., to 0,65 deaths per 1000 live births, thus representing a decrease of $53 \%$ over 10 years. (18) 
The mechanisms by which the prone sleep position may lead to death remain uncertain. However, it has been found that prone-sleeping infants adopt the facedown position either spontaneously or in response to noxious stimulus. In infants sleeping in a facedown position, the concentration of inspired carbon dioxide, and of the expired end-tidal carbon dioxide both rise.
(19) Lethal rebreathing is compatible with the aforementioned theoretical mechanisms of SIDS. Compensatory mechanisms to hypercapnia and hypoxia are hyperventilation and arousal responses. However, the impaired alveolar ventilation and significantly higher hypercarbic arousal thresholds have been found in some infants with ALTE. (20) Furthermore, the ventilatory response to hypercarbia can be blunted in infants who experience repeated episodes of hypercarbia due to a constant prone sleeping position on a soft mattress. (21) It may be that rebreathing in association with deficient arousal responses to hypoxemia and hypercapnia might explain the reason why the SIDS-infants did not awaken and gain access to fresh air.

\section{REFERENCES}

1. Consensuns statement, National Institutes of Health Consensus Conference on Infantile Apnea and Home Monitoring, Sept 29 to Oct 1 , 1986. Pediatrics 1987;79:292-9.

2. Willinger M, James LS, Catz C. Defining the sudden infant death syndrome (SIDS): deliberations on an expert panel convened by the National Institute of Child Health and Human Development. Pediatr Pathol 1991;11:677-84.

3. Brooks JG. Apparent life-threatening events and apnea of infancy. Clin Perinatol 1992;19:809-38.

4. Oren J, Kelly D, Shannon DC. Identification of a high-risk group for sudden-death syndrome among infants who were resusctitated for sleep apnea. Pediatrics 1986;77:495-9.

5. Consensuns statement, National Institutes of Health Consensus Conference on Infantile Apnea and Home Monitoring, Sept 29 to Oct 1 , 1986. Pediatrics 1987;79:292-9.

6. Kopp N, Eymin C, Denory L, Marti D, Jordan D. Pathology and biochemistry of the central nervous system in sudden infant death syndrome: a short review. Acta Paediatr 1993;389(suppl):86-7.

7. Sawaguchi T, Patricia F, Kadhim H, Groswasser J, Sottiaux M, Nishida H, et al, editors. Clinicopathological correlation between brainstem gliosis using GFAP as a marker of sleep apnea in sudden infant death syndrome. Early Human Deve 2003:75(suppl):3-11.

8. Kahn A, Groswasser J, Sottiaux M, Clinical problems in relation to apparent life-threatening events in infants. Acta Paediatr 1993;389(suppl):107-10.

9. White M, Beckett M, O'Regan M, Autonomic function in SIDS. Acta Paediatr 1993;389(suppl):105-6.

10. Sridhar R, Thach BT, Kelly DH, Henslee JA. Characterization of successful and failed autoresuscitation in human infants, including those dying of SIDS. Pediatr Pulmonol 2003;36:113-22.

11. Franco P, Seret N, Van Hees JN, Scaillet S. Vermeulen F. Groswasser J. et al, editors. Decreased arousals among healthy infants after shortterm sleep deprivation. Pediatrics 2004;114:e192-e7.

12. Sawnani H, Jackson T, Murphy T, Beckerman R. Simakajornboon N. The effects of maternal smoking on respiratory and arousal patterns in preterm infants during sleep. Am J Respir Crit Care Med 2004;169:733-8.

13. Feldman JL, Michell GS, Natie EE. Breathing: rhythmicity, plasticity, chemosensitivity. Annu Rev Neurosci 2003;26:239-66.

14. Nattie EE, Prabhakar NR. Peripheral and central chemosensitivity: multiple mechanisms, multiple sites? A workshop summary. Adv Exp Med Biol 2001;499:73-80.

15. Waters KA, Gozal D. Responses to hypoxia during early development. Respir Physiol Neurobiol 2003;136:115-29.

16. Gozal A, Gozal D. Respiratory plasticity following intermittent hypoxia: developmental interactions. J Appl Physiol 2001;90:1995-9.

17. Durand E, Lofaso F, Dauger S, Vardon G, Gaultier C, Gallego J. Intermittent hypoxia induces transient arousal delay in newborn mice. J Appl Physiol 2006:96:1216-22.

18. AAP. Task force on Sudden infant death syndrome. The changing concept of sudden infant death syndrome: diagnostic, coding shifts, controversies regarding the sleeping environment, and new variables to consider in reducing risk. Pediatrics 2005;116:1245-55.

19. Chiodini BA, Bradley TT. Impaired ventilation in infants sleeping facedown: potential significance for sudden infant death syndrome. J Pediatr 1993;123:686-92.

20. McCulloch K, Brouillette RT, Guzzetta AJ, Hunt CE. Arousal response in near-miss sudden infant death syndrome and normal infants. J Pediatr 1982;101:911-7.

21. Skadberg BT, Oterhals A, Finbourd K, Markestad T. CO2 rebreathing: a possible contributory factor to some cases of sudden infant death. Acta Paed 1995;84:988-95 